\title{
Orexin/hypocretin (Orx/Hcrt) transmission and drug-seeking behavior: is the paraventricular nucleus of the thalamus (PVT) part of the drug seeking circuitry?
}

\author{
Rémi Martin-Fardon ${ }^{1 *}$ and Benjamin Boutrel ${ }^{2}$ \\ ${ }^{1}$ Molecular and Integrative Neurosciences Department, The Scripps Research Institute, La Jolla, CA, USA \\ 2 Department of Psychiatry, Center for Psychiatric Neuroscience, Lausanne University Hospital, Lausanne, Switzerland
}

Edited by:

Luis De Lecea, Stanford University

School of Medicine, USA

Reviewed by:

Gregg Stanwood, Vanderbilt

University, USA

Garret Stuber, University of North

Carolina at Chapel Hill, USA

\section{*Correspondence:}

Rémi Martin-Fardon, Molecular and Integrative Neurosciences

Department, The Scripps Research

Institute, 10550 North Torrey Pines

Road, SP30-2120, La Jolla,

CA 92037, USA.

e-mail: rmartinf@scripps.edu
The orexin/hypocretin (Orx/Hcrt) system has long been considered to regulate a wide range of physiological processes, including feeding, energy metabolism, and arousal. More recently, concordant observations have demonstrated an important role for these peptides in the reinforcing properties of most drugs of abuse. Orx/Hcrt neurons arise in the lateral hypothalamus (LH) and project to all brain structures implicated in the regulation of arousal, stress, and reward. Although Orx/Hcrt neurons have been shown to massively project to the paraventricular nucleus of the thalamus (PVT), only recent evidence suggested that the PVT may be a key relay of Orx/Hcrt-coded reward-related communication between the LH and both the ventral and dorsal striatum. While this thalamic region was not thought to be part of the "drug addiction circuitry," an increasing amount of evidence demonstrated that the PVT—particularly PVT Orx/Hcrt transmission—was implicated in the modulation of reward function in general and several aspects of drug-directed behaviors in particular. The present review discusses recent findings that suggest that maladaptive recruitment of PVT Orx/Hcrt signaling by drugs of abuse may promote persistent compulsive drug-seeking behavior following a period of protracted abstinence and as such may represent a relevant target for understanding the long-term vulnerability to drug relapse after withdrawal.

Keywords: paraventricular nucleus of the thalamus, orexin, hypocretin, drug-seeking behavior, natural reward

\section{INTRODUCTION}

Drug addiction is a chronically relapsing disorder characterized by compulsive drug seeking and use (O'Brien and McLellan, 1996; Leshner, 1997; O’Brien et al., 1998; McLellan et al., 2000). Advances have been made in elucidating the neurocircuitry that mediates craving and drug seeking. Functional brain imaging in humans (e.g., Miller and Goldsmith, 2001; Goldstein and Volkow, 2002; Daglish and Nutt, 2003) and brain site-specific manipulations in animals (e.g., Everitt et al., 2001; Cardinal et al., 2002; See, 2002; Weiss, 2005) implicate interconnected cortical and limbic brain regions in response to drug cue-, drug priming-, and stress-induced reinstatement. Major components of this circuitry include the medial prefrontal cortex (mPFC), basolateral amygdala (BLA), central nucleus of the amygdala (CeA), bed nucleus of the stria terminalis (BNST), hippocampus, nucleus accumbens (NAC), and, more recently, dorsal striatum, which is thought to participate in consolidating stimulus-response habits via the engagement of corticostriatal loops (Everitt et al., 2001; McFarland and Kalivas, 2001; Ito et al., 2002; Kalivas and Volkow, 2005; Belin and Everitt, 2008; Steketee and Kalivas, 2011). However, unclear is what differentiates neural signaling related to normal appetitive behavior $v s$. compulsive behavior that results from long-term drug exposure. There is an overlap between the brain regions implicated in the processing of natural rewards and drugs of abuse, and it is thought that neural circuitry encoded for natural rewards is usurped by drugs of abuse. Neuroplasticity within this neural circuitry is believed to be responsible for the maladaptive (compulsive) behavior characteristic of addiction (Kelley and Berridge, 2002; Aston-Jones and Harris, 2004; Kalivas and O'Brien, 2008; Wanat et al., 2009), which may account for the interindividual vulnerability to drug abuse.

\section{RECRUITMENT OF THE Orx/Hcrt SYSTEM BY DRUGS OF ABUSE}

About 10 years ago, the orexin/hypocretin (Orx/Hcrt) system, already known to regulate a wide range of physiological processes, was shown to be recruited by drugs of abuse. Indeed, orexin A (Orx-A or hypocretin-1 [Hcrt-1]) and orexin B (Orx-B or hypocretin-2 [Hcrt-2]) were initially considered hypothalamic neuropeptides that regulate feeding, energy metabolism (Sakurai et al., 1998; Edwards et al., 1999; Haynes et al., 2000, 2002; Willie et al., 2001; Teske et al., 2010), and arousal (Sutcliffe and de Lecea, 2002; Taheri et al., 2002). Among the two Orx/Hcrt receptors identified, Hcrt-r1 binds to Orx-A/Hcrt-1 with 20-30 nM affinity but has much lower affinity (10-1000-fold lower) for OrxB/Hcrt-2, and Hcrt-r2 binds to both peptides with similar affinity (in the $40 \mathrm{nM}$ range; Sakurai et al., 1998; Ammoun et al., 2003; Scammell and Winrow, 2011). Orx/Hcrt cell bodies are essentially found in the lateral hypothalamus (LH), a brain region long associated with reward and motivation (for review, see DiLeone et al., 2003), perifornical hypothalamus (PFA), and dorsomedial hypothalamus (DMH). Hypothalamic Orx/Hcrt neurons project 
to brainstem nuclei where they are considered to play a major role in the regulation of arousal and modulation of stress responses (Baldo et al., 2003; Winsky-Sommerer et al., 2004). They also project to the paraventricular nucleus of the thalamus (PVT), NAC shell (NACsh), ventral pallidum (VP), ventral tegmental area (VTA), CeA, and BNST (Peyron et al., 1998; Baldo et al., 2003). A past conjecture suggested a dichotomy in Orx/Hcrt function, with Orx/Hcrt neurons in the LH regulating reward processes and Orx/Hcrt neurons in the PFA and DMH being mostly involved in the regulation of arousal and stress responses (Harris and Aston-Jones, 2006). However, recent evidence opposes this interpretation because similar patterns of Fos-positive Orx/Hcrt cells were observed in both the PFA/LH and DMH in rats exposed to contextual stimuli previously paired with ethanol availability (Dayas et al., 2008). Furthermore, medial and lateral Orx/Hcrt cells were shown to project to both the locus coeruleus and VTA, confirming that convergent projections from different Orx/Hcrt populations to these two brain areas may strengthen the temporal association between stress, arousal, and reward-seeking, thus optimizing goal-oriented behavioral strategies (Calipari and Espana, 2012; Gonzalez et al., 2012).

In addition to their involvement in the regulation of natural rewards, recent evidence showed that hypothalamic Orx/Hcrt neurons played a significant role in the modulation of reward function and, particularly, drug-directed behaviors (Harris et al., 2005). Hypothalamic Orx/Hcrt neurons become activated by stimuli associated with food, morphine, cocaine, and ethanol (Harris et al., 2005; Dayas et al., 2008; Martin-Fardon et al., 2010; Jupp et al., 2011). Similarly, the expression of conditioned place preference induced not only by food but also by morphine and cocaine is associated with activation of Orx/Hcrt neurons in the LH (Harris et al., 2005) likely due to the stimulation of LH Orx/Hcrt by rostral lateral septum afferents (Sartor and Aston-Jones, 2012). Consistent with these observations, intraVTA microinjection of Orx-A produces a renewal of morphineinduced conditioned place preference, whereas administration of the Hcrt-r1 antagonist $N$-(2-methyl-6-benzoxazolyl)- $N^{\prime}-1,5$ n-aphthyridin-4-yl urea (SB334867) decreases the expression of morphine-induced conditioned place preference (Harris et al., 2005). SB334867 also blocks the acquisition of cocaine-induced behavioral sensitization and potentiation of excitatory currents induced by cocaine in VTA dopamine neurons (Borgland et al., 2006). Intra-VTA administration of SB334867 reduces the motivation to self-administer cocaine and attenuates the cocaine-induced enhancement of dopamine signaling in the NAC (Espana et al., 2010). Blockade of Hcrt-r1 decreases ethanol (Lawrence et al., 2006) and nicotine (Hollander et al., 2008) self-administration, inhibits cue-induced reinstatement of ethanol (Lawrence et al., 2006) and cocaine (Smith et al., 2010) seeking, and attenuates stress-induced reinstatement of cocaine and ethanol seeking (Boutrel et al., 2005; Richards et al., 2008).

Thus, behavioral and functional evidence indicates a role for Orx/Hcrt signaling in the motivational effects of cocaine and other drugs of abuse (Borgland et al., 2006; Bonci and Borgland, 2009; Thompson and Borgland, 2011), but questions remain about what differentiates Orx/Hcrt signaling related to normal appetitive behavior $v s$. compulsive behavior that results from long-term drug exposure.

\section{DIFFERENTIAL RECRUITMENT OF THE Orx/Hcrt SYSTEM BY DRUGS OF ABUSE AND NATURAL REWARDS}

One hypothesis concerning the control of drug-seeking behavior is that the neural circuits that mediate these effects are common motivational circuits that are more robustly activated by drug-related stimuli and not specific to addiction-related events. This activation that normally governs responding for natural rewards creates new motivational states or tilts processes that normally govern responding for natural rewards toward drugdirected behavior (Kelley and Berridge, 2002). Considered to orchestrate the appropriate levels of alertness required for the elaboration and execution of goal-oriented behaviors, Orx/Hcrt is one legitimate candidate for further investigating how a system normally involved in the regulation of motivation and arousal may trigger a pathological state that elicits compulsive craving and relapse to drug seeking (Boutrel et al., 2010).

Evidence has accumulated that the Orx/Hcrt system is, in fact, more strongly engaged by drugs of abuse compared with natural non-drug reinforcers. For example, it has been shown that SB334867 treatment significantly reduced responding for ethanol but not sucrose under a progressive-ratio schedule of reinforcement (Jupp et al., 2011). An even more striking observation was that, although the stimuli conditioned to cocaine, ethanol, and a conventional reinforcer were shown to equally elicit reinstatement, SB334867 treatment selectively reversed conditioned reinstatement induced by a cocaine- or ethanol-related stimulus but had no effects on the same stimulus conditioned to a conventional reinforcer (sweetened condensed milk [SCM] or SuperSac [3\%/0.125\%, w/v, glucose/saccharin]; Martin-Fardon and Weiss, 2009, 2012; Martin-Fardon et al., 2010).

The pharmacological and neural mapping data are difficult to reconcile with the role of the Orx/Hcrt system in behavior motivated by food (i.e., a natural reward) and its more recently discovered role in drug reward. One hypothesis concerning the control of drug-seeking behavior is that the neural circuits that mediate the effects of drug cues are not specific to addictionrelated events but rather are common motivational circuits that are more robustly activated by drug-related stimuli. This activation will create new motivational states or tilt processes that normally govern responding for natural rewards toward drugdirected behavior (Kelley and Berridge, 2002). Drugs of abuse may produce this effect by neuroadaptively altering the neural systems that regulate motivation directed toward natural rewards. Evidence of drug-induced dysregulation of the Orx/Hcrt system exists for alcohol. For example, prepro-orexin mRNA is up-regulated in the $\mathrm{LH}$ in inbred alcohol-preferring (iP) rats following chronic ethanol consumption (Lawrence et al., 2006). A possibility derived from this hypothesis is that the Orx/Hcrt system may, over the course of repeated drug use, acquire a preferential role in mediating the effects of stimuli conditioned to drugs of abuse $v s$. natural rewards. Consequently, one explanation for the preferential role of SB334867 in conditioned reinstatement for drugs $v s$. non-drugs could be that drugs neuroadaptively alter the neural systems that regulate motivation normally directed 
toward natural rewards that is revealed by pharmacological (e.g., SB334867) manipulations.

Maladaptive recruitment of the Orx/Hcrt system by drugs of abuse is also suggested by findings that described neuroadaptive changes within the VTA. For example, voluntary cocaine and natural reward self-administration induces a common, shortlasting neuroadaptation in VTA dopaminergic neurons (i.e., increased glutamatergic function; Chen et al., 2008). However, this enhanced synaptic strength persists and is resistant to extinction in rats that self-administer cocaine only and not in rats that self-administer a non-drug reinforcer (Chen et al., 2008). Interestingly, several lines of evidence suggest that the participation of the VTA in cocaine-induced neuronal and behavioral changes requires Orx/Hcrt inputs. For example, activation of Hcrt-r1 in the VTA is necessary for the development of cocaineinduced locomotor sensitization (Borgland et al., 2006), and Orx-A/Hcrt-1-mediated N-methyl-D-aspartate (NMDA) receptor plasticity in the VTA is increased in rats that self-administer cocaine (Borgland et al., 2009). Additionally, short-lasting neuroadaptations in VTA dopaminergic neurons induced by high-fat chocolate food pellets have been described (Borgland et al., 2009), suggesting that the Orx/Hcrt-VTA system initially participates in the regulation of the motivation to obtain potent reinforcers in general (i.e., drug or highly palatable food). In contrast, drug-induced neuroadaptation of the Orx/Hcrt-VTA system is long-lasting, an effect that may be linked to the possible tilting of this system toward promoting and controlling drug-directed behavior.

\section{IMPLICATION OF Orx/Hcrt-PVT TRANSMISSION IN MALADAPTIVE (DRUG-SEEKING) BEHAVIOR}

Anatomically, it has been shown that the PVT is the target of numerous hypothalamic peptides involved in energy homeostasis (Freedman and Cassell, 1994; Otake, 2005), including Orx/Hcrt (Kirouac et al., 2005, 2006; Ishibashi et al., 2005). It is believed that the PVT plays a key role in energy homeostasis, arousal, temperature modulation, endocrine regulation, and reward (Bhatnagar and Dallman, 1998, 1999; Van Der Werf et al., 2002; Kelley et al., 2005; Parsons et al., 2006). Specifically and particularly important for this review, lesions of the PVT were shown to increase feeding behavior and body weight (Bhatnagar and Dallman, 1999) while attenuating the increases in both locomotor activity and blood corticosterone levels normally seen during the anticipation of food reward (Nakahara et al., 2004). Furthermore, the PVT was shown to be critically involved in mediating the effects of Orx/Hcrt on brain dopamine levels and reward-based feeding behaviors (Choi et al., 2012). These findings strongly suggest an important role for Orx/Hcrt-PVT signaling in food intake regulation.

A major Orx/Hcrt projection exists from the LH/PFA to the PVT (Kirouac et al., 2005; Parsons et al., 2006), and the PVT has been proposed to be a key relay (see Figure 1), gating Orx/Hcrtcoded reward-related communication between the LH/PFA and both the ventral and dorsal striatum (Kelley et al., 2005). This "hypothalamic-thalamic-striatal axis" may have evolved to prolong central motivational states and promote feeding beyond the fulfillment of immediate energy needs, thereby creating energy

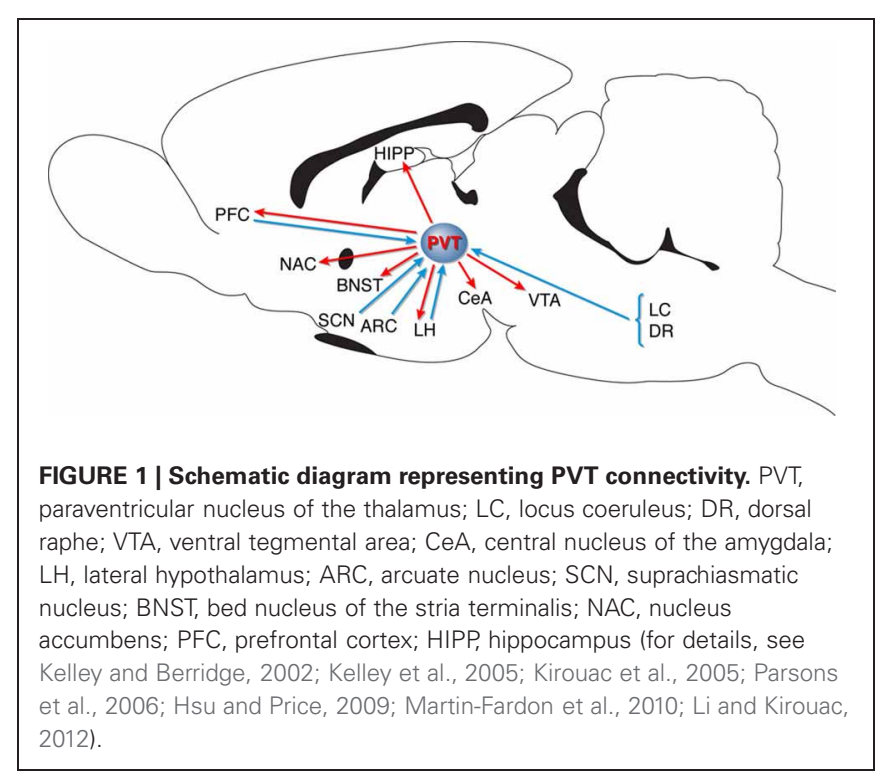

reserves for potential future food shortages (Kelley et al., 2005). With regard to "drug seeking-related brain regions", it is important to note that the PVT specifically projects to the CeA, BNST, NAC, VTA, and hippocampus (e.g., Kelley and Berridge, 2002; Kelley et al., 2005; Parsons et al., 2006; Hsu and Price, 2009; Martin-Fardon et al., 2010). Finally, recent data have shown that the PVT receives projections from the PFC, suggesting that these connections could modulate the expression of emotional behaviors (Li and Kirouac, 2012).

Although not usually thought of as part of drug-seeking neurocircuitry, direct and indirect findings recently supported a role for Orx/Hcrt-PVT signaling in drug-oriented behaviors. First, acute nicotine treatment was shown to activate Orx/Hcrt neurons that project to the basal forebrain and PVT, supporting a role for the Orx/Hcrt system in mediating certain aspects of nicotine-elicited wakefulness rather than proving a role for Orx/Hcrt-PVT signaling in tobacco addiction (Pasumarthi and Fadel, 2008), but such a link cannot be underestimated until proven false. Second, Orx/Hcrt peptides within the PVT have been suggested to regulate negative emotional states ( $\mathrm{Li}$ et al., 2010a,b). Orx/Hcrt-PVT signaling was also shown to be critically involved in the expression of conditioned place aversion to morphine withdrawal (Li et al., 2011). These latter two observations may support a role for Orx-Hcrt signaling within the PVT in the negative emotional state that is putatively responsible for triggering the urge to seek drug during withdrawal of after a period of abstinence. A more direct observation confirmed that drug-related contextual cues activate Orx/Hcrt neurons (Dayas et al., 2008). Indeed, significantly larger numbers of Fos-positive hypothalamic Orx/Hcrt neurons were seen in rats exposed to contextual stimuli previously associated with ethanol availability compared with rats exposed to the same stimulus previously paired with non-reward. Moreover, presentation of the ethanol-related stimuli also increased the number of Fos-positive PVT neurons, and these neurons were closely associated with Orx/Hcrt fibers (for additional details, see Dayas et al., 2008). 
Other evidence supports a role for Orx/Hcrt projections from the LH to PVT in regulating drug-seeking behavior. Recent data confirmed that context-induced reinstatement of alcoholic beer seeking is associated with recruitment of a PVT-ventral striatum pathway and that excitotoxic lesion of this structure (Hamlin et al., 2009) or discrete administration of a $\kappa$ opioid receptor agonist (Marchant et al., 2010) prevents context-induced reinstatement of alcohol seeking. In conclusion, it is hypothesized that maladaptive recruitment of the Orx/Hcrt system by drugs of abuse may tilt its function toward excessive drug-directed behavior, which may explain the increased sensitivity of this peptidergic system to antagonist interference with drug-seeking behavior as opposed to behavior directed toward natural rewards.

Furthermore, it was recently shown that although Orx/Hcrt microinjections into the PVT exerted a priming-like effect, reinstating both extinguished cocaine- and SCM-seeking behavior, Orx/Hcrt produced (1) two different dose-response functions for cocaine seeking $v s$. SCM seeking and (2) a stronger reinstatement of cocaine seeking vs. SCM seeking at moderate doses (MartinFardon et al., 2011). This observation suggests of a leftward shift of the Orx-A/Hcrt-1 dose-response curve in cocaine-experienced animals, implying that cocaine produced a dysregulation of Orx/Hcrt-PVT transmission that is revealed following exogenous Orx/Hcrt administration. Moreover, recent findings have demonstrated that discrete inactivation of the PVT with tetrodotoxin (TTX) prevented cocaine priming-induced reinstatement (James et al., 2010), further implicating this thalamic structure in drugseeking behavior, although the same researchers claimed that Orx/Hcrt-1 receptor signaling within the VTA but not PVT was critical in the regulation of cue-induced reinstatement of cocaine-seeking behaviors (James et al., 2011).

\section{CONCLUSION/PERSPECTIVES}

Currently, the available therapeutic approaches fail to completely treat and address the compulsive nature of drug seeking and drug taking associated with addiction. Evidence indicates that dysfunctional Orx/Hcrt transmission contributes to drug seeking vs. natural reward seeking, and an increasing amount of data has

\section{REFERENCES}

Ammoun, S., Holmqvist, T., Shariatmadari, R., Oonk, H. B., Detheux, M., Parmentier, M., et al. (2003). Distinct recognition of OX1 and OX2 receptors by orexin peptides. J. Pharmacol. Exp. Ther. 305, 507-514.

Aston-Jones, G., and Harris, G. C. (2004). Brain substrates for increased drug seeking during protracted withdrawal. Neuropharmacology 47(Suppl. 1), 167-179.

Aujla, H., Martin-Fardon, R., and Weiss, F. (2008). Rats with extended access to cocaine exhibit increased stress reactivity and sensitivity to the anxiolytic-like effects of the mGluR 2/3 agonist
LY379268 during abstinence. Neuropsychopharmacology 33, 1818-1826.

Baldo, B. A., Daniel, R. A., Berridge, C. W., and Kelley, A. E. (2003). Overlapping distributions of orexin/hypocretin- and dopaminebeta-hydroxylase immunoreactive fibers in rat brain regions mediating arousal, motivation, and stress. J. Comp. Neurol. 464, 220-237.

Belin, D., and Everitt, B. J. (2008). Cocaine seeking habits depend upon dopamine-dependent serial connectivity linking the ventral with the dorsal striatum. Neuron 57, 432-441.

Bhatnagar, S., and Dallman, M. (1998). Neuroanatomical basis for facilitation of hypothalamic-

now identified the PVT, a brain region not usually included in the neurocircuitry of addiction, to be recruited by drugs of abuse, opening up a new area of targets for efficient pharmacotherapy.

Notably, however, drug addiction is often associated with increased drug consumption that can modify the pharmacological profile of promising therapeutic agents, possibly resulting in drug-induced neuroadaptation (for review, see Kalivas and O'Brien, 2008; Moussawi et al., 2009). For instance, following extended-access cocaine self-administration (6h/day), it was shown that (-)-2-oxa-4-aminobicylco[3.1.0]hexane4,6-dicarboxylic acid (LY379268), a metabotropic glutamate receptor (mGluR) 2/3 agonist, became more efficient at preventing anxiety-like behavior and decreasing cocaine selfadministration (Aujla et al., 2008; Hao et al., 2010), whereas the effects of an mGluR5 antagonist, 3-[(2-methyl-1,3-thiazol-4yl)ethynyl]piperidine (MTEP), were blunted (Hao et al., 2010). A similar behavioral pharmacological profile was observed in animals that had a history of alcohol dependence, in which LY379268 and MTEP dose-dependently reduced both alcohol self-administration and the reinstatement of alcohol seeking induced by footshock stress, but LY379268 was more effective than MTEP in inhibiting both behaviors in postdependent animals compared with non-dependent animals (Sidhpura et al., 2010). Consequently, considering the importance of relapse prevention in postdependent individuals, important issues that require further research are to identify (1) whether Orx/HcrtPVT transmission becomes further dysfunctional following dependence and (2) the most effective pharmacological tools (i.e., Hcrt-r antagonists) for relapse and craving prevention in postdependent individuals.

\section{ACKNOWLEDGMENTS}

This is publication number 21836 from The Scripps Research Institute. This research was supported by NIH/NIDA grant DA033344 (Rémi Martin-Fardon) and Swiss National Science Foundation grants 3100A0-112101 and 3100A0-133056 (Benjamin Boutrel). The authors thank G. Cauvi and M. Arends for assistance with the preparation of the manuscript.

pituitary-adrenal responses to a novel stressor after chronic stress. Neuroscience 84, 1025-1039.

Bhatnagar, S., and Dallman, M. F. (1999). The paraventricular nucleus of the thalamus alters rhythms in core temperature and energy balance in a statedependent manner. Brain Res. 851, 66-75.

Bonci, A., and Borgland, S. (2009). Role of orexin/hypocretin and CRF in the formation of drug-dependent synaptic plasticity in the mesolimbic system. Neuropharmacology 56(Suppl. 1), 107-111.

Borgland, S. L., Chang, S. J., Bowers, M. S., Thompson, J. L., Vittoz, N., Floresco, S. B., et al. (2009). Orexin A/hypocretin-1 selectively promotes motivation for positive reinforcers. J. Neurosci. 29, 11215-11225.

Borgland, S. L., Taha, S. A., Sarti, F. Fields, H. L., and Bonci, A. (2006). Orexin A in the VTA is critical for the induction of synaptic plasticity and behavioral sensitization to cocaine. Neuron 49, 589-601.

Boutrel, B., Cannella, N., and De Lecea, L. (2010). The role of hypocretin in driving arousal and goaloriented behaviors. Brain Res. 1314, 103-111.

Boutrel, B., Kenny, P. J., Specio, S. E., Martin-Fardon, R., Markou, A., Koob, G. F., et al. (2005). Role for hypocretin in mediating stressinduced reinstatement of cocaineseeking behavior. Proc. Natl. Acad. Sci. U.S.A. 102, 19168-19173. 
Calipari, E. S., and Espana, R. A. (2012). Hypocretin/orexin regulation of dopamine signaling: implications for reward and reinforcement mechanisms. Front. Behav. Neurosci. 6:54. doi: 10.3389/fnbeh.2012.00054

Cardinal, R. N., Parkinson, J. A., Hall, J., and Everitt, B. J. (2002). Emotion and motivation: the role of the amygdala, ventral striatum, and prefrontal cortex. Neurosci. Biobehav. Rev. 26, 321-352.

Chen, B. T., Bowers, M. S., Martin, M., Hopf, F. W., Guillory, A. M., Carelli, R. M., et al. (2008). Cocaine but not natural reward self-administration nor passive cocaine infusion produces persistent LTP in the VTA. Neuron 59, 288-297.

Choi, D. L., Davis, J. F., Magrisso, I. J., Fitzgerald, M. E., Lipton, J. W., and Benoit, S. C. (2012). Orexin signaling in the paraventricular thalamic nucleus modulates mesolimbic dopamine and hedonic feeding in the rat. Neuroscience 210, 243-248.

Daglish, M. R., and Nutt, D. J. (2003). Brain imaging studies in human addicts. Eur. Neuropsychopharmacol.

13 , 453-458.

Dayas, C. V., McGranahan, T. M., Martin-Fardon, R., and Weiss, F. (2008). Stimuli linked to ethanol availability activate hypothalamic CART and orexin neurons in a reinstatement model of relapse. Biol. Psychiatry 63, 152-157.

DiLeone, R. J., Georgescu, D., and Nestler, E. J. (2003). Lateral hypothalamic neuropeptides in reward and drug addiction. Life Sci. 73, 759-768.

Edwards, C. M., Abusnana, S., Sunter, D., Murphy, K. G., Ghatei, M. A., and Bloom, S. R. (1999). The effect of the orexins on food intake: comparison with neuropeptide $\mathrm{Y}$, melanin-concentrating hormone and galanin. J. Endocrinol. 160, R7-R12.

Espana, R. A., Oleson, E. B., Locke, J. L., Brookshire, B. R., Roberts, D. C., and Jones, S. R. (2010). The hypocretin-orexin system regulates cocaine self-administration via actions on the mesolimbic dopamine system. Eur. J. Neurosci. 31, 336-348.

Everitt, B. J., Dickinson, A., and Robbins, T. W. (2001). The neuropsychological basis of addictive behaviour. Brain Res. Brain Res. Rev. $36,129-138$

Freedman, L. J., and Cassell, M. D. (1994). Relationship of thalamic basal forebrain projection neurons to the peptidergic innervation of the midline thalamus. J. Comp. Neurol. 348, 321-342.

Goldstein, R. Z., and Volkow, N. D. (2002). Drug addiction and its underlying neurobiological basis: neuroimaging evidence for the involvement of the frontal cortex. Am. J. Psychiatry 159, 1642-1652.

Gonzalez, J. A., Jensen, L. T., Fugger, L., and Burdakov, D. (2012). Convergent inputs from electrically and topographically distinct orexin cells to locus coeruleus and ventral tegmental area. Eur. J. Neurosci. 35, 1426-1432.

Hamlin, A. S., Clemens, K. J., Choi, E. A., and McNally, G. P. (2009). Paraventricular thalamus mediates context-induced reinstatement (renewal) of extinguished reward seeking. Eur. J. Neurosci. 29, 802-812.

Hao, Y., Martin-Fardon, R., and Weiss, F. (2010). Behavioral and functional evidence of metabotropic glutamate receptor $2 / 3$ and metabotropic glutamate receptor 5 dysregulation in cocaine-escalated rats: factor in the transition to dependence. Biol. Psychiatry 68, 240-248.

Harris, G. C., and Aston-Jones, G. (2006). Arousal and reward: a dichotomy in orexin function. Trends Neurosci. 29, 571-577.

Harris, G. C., Wimmer, M., and AstonJones, G. (2005). A role for lateral hypothalamic orexin neurons in reward seeking. Nature 437, 556-559.

Haynes, A. C., Chapman, H., Taylor C., Moore, G. B., Cawthorne, M. A., Tadayyon, M., et al. (2002). Anorectic, thermogenic and antiobesity activity of a selective orexin1 receptor antagonist in ob/ob mice. Regul. Pept. 104, 153-159.

Haynes, A. C., Jackson, B., Chapman, H., Tadayyon, M., Johns, A., Porter, R. A., et al. (2000). A selective orexin-1 receptor antagonist reduces food consumption in male and female rats. Regul. Pept. 96, 45-51.

Hollander, J. A., Lu, Q., Cameron, M. D., Kamenecka, T. M., and Kenny, P. J. (2008). Insular hypocretin transmission regulates nicotine reward. Proc. Natl. Acad. Sci. U.S.A. 105, 19480-19485.

Hsu, D. T., and Price, J. L. (2009). Paraventricular thalamic nucleus: subcortical connections and innervation by serotonin, orexin, and corticotropin-releasing hormone in macaque monkeys. J. Comp. Neurol. $512,825-848$.

Ishibashi, M., Takano, S., Yanagida, H., Takatsuna, M., Nakajima, K., Oomura, Y., et al. (2005). Effects of orexins/hypocretins on neuronal activity in the paraventricular nucleus of the thalamus in rats in vitro. Peptides 26, 471-481.

Ito, R., Dalley, J. W., Robbins, T. W. and Everitt, B. J. (2002). Dopamine release in the dorsal striatum during cocaine-seeking behavior under the control of a drug-associated cue. J. Neurosci. 22, 6247-6253.

James, M. H., Charnley, J. L., Jones, E., Levi, E. M., Yeoh, J. W., Flynn, J. R., et al. (2010). Cocaineand amphetamine-regulated transcript (CART) signaling within the paraventricular thalamus modulates cocaine-seeking behaviour. PLoS ONE 5:e12980. doi: 10.1371/journal.pone.0012980

James, M. H., Charnley, J. L., Levi, E. M., Jones, E., Yeoh, J. W. Smith, D. W., et al. (2011). Orexin1 receptor signalling within the ventral tegmental area, but not the paraventricular thalamus, is critical to regulating cue-induced reinstatement of cocaine-seeking. Int. J. Neuropsychopharmacol. 14 684-690.

Jupp, B., Krivdic, B., Krstew, E., and Lawrence, A. J. (2011). The orexin receptor antagonist SB-334867 dissociates the motivational properties of alcohol and sucrose in rats. Brain Res. 1391, 54-59.

Jupp, B., Krstew, E., Dezsi, G., and Lawrence, A. J. (2011). Discrete cueconditioned alcohol-seeking after protracted abstinence: pattern of neural activation and involvement of orexin receptors. Br. J. Pharmacol. $162,880-889$.

Kalivas, P. W., and O'Brien, C. (2008) Drug addiction as a pathology of staged neuroplasticity. Neuropsychopharmacology 33 166-180.

Kalivas, P. W., and Volkow, N. D (2005). The neural basis of addiction: a pathology of motivation and choice. Am. J. Psychiatry 162 1403-1413.

Kelley, A. E., Baldo, B. A., and Pratt, W. E. (2005). A proposed hypothalamic-thalamic-striatal axis for the integration of energy balance, arousal, and food reward. $J$. Comp. Neurol. 493, 72-85.

Kelley, A. E., and Berridge, K. C (2002). The neuroscience of natural rewards: relevance to addictive drugs. J. Neurosci. 22, 3306-3311.

Kirouac, G. J., Parsons, M. P., and Li, S. (2005). Orexin (hypocretin) innervation of the paraventricular nucleus of the thalamus. Brain Res. 1059, 179-188.

Kirouac, G. J., Parsons, M. P., and $\mathrm{Li}, \quad$ S. (2006). Innervation of the paraventricular nucleus of the thalamus from cocaine- and amphetamine-regulated transcript (CART) containing neurons of the hypothalamus. J. Comp. Neurol. 497, 155-165.

Lawrence, A. J., Cowen, M. S., Yang, H. J., Chen, F., and Oldfield, B. (2006). The orexin system regulates alcoholseeking in rats. Br. J. Pharmacol. 148, 752-759.

Leshner, A. I. (1997). Addiction is a brain disease, and it matters. Science $278,45-47$.

Li, S., and Kirouac, G. J. (2012). Sources of inputs to the anterior and posterior aspects of the paraventricular nucleus of the thalamus. Brain Struct. Funct. 217, 257-273.

Li, Y., Li, S., Wei, C., Wang, H., Sui, N., and Kirouac, G. J. (2010a). Changes in emotional behavior produced by orexin microinjections in the paraventricular nucleus of the thalamus. Pharmacol. Biochem. Behav. 95, 121-128.

Li, Y., Li, S., Wei, C., Wang, H., Sui, N., and Kirouac, G. J. (2010b). Orexins in the paraventricular nucleus of the thalamus mediate anxiety-like responses in rats. Psychopharmacology (Berl.) 212, 251-265.

Li, Y., Wang, H., Qi, K., Chen, X., Li, S., Sui, N., et al. (2011). Orexins in the midline thalamus are involved in the expression of conditioned place aversion to morphine withdrawal. Physiol. Behav. 102, 42-50.

Marchant, N. J., Furlong, T. M., and McNally, G. P. (2010). Medial dorsal hypothalamus mediates the inhibition of reward seeking after extinction. J. Neurosci. 30, 14102-14115.

Martin-Fardon, R., Leos, B. N., Kerr, T. M., and Weiss, F. (2011). Administration of Orexin/Hypocretin (Orx/Hcrt) in the Paraventricular Nucleus of the Thalamus (PVT) Produces CocaineSeeking: Comparison with Natural Reward-Seeking. Program No. 69.10. 2011 Neuroscience Meeting Planner. Washington, DC: Society for Neuroscience. [Online].

Martin-Fardon, R., and Weiss, $\mathrm{F}$ (2009). Differential Effects of an Orx/Hcrt Antagonist on Reinstatement Induced by a Cue Conditioned to Cocaine vs. Palatable Natural Reward. Program No. 65.21. 2009 Neuroscience Meeting Planner, Chicago, IL: Society for Neuroscience. [Online].

Martin-Fardon, R., and Weiss, F. (2012). N-(2-methyl-6-ben zoxazolyl)-N' $-1, \quad 5$-naphthyridin4-yl urea (SB334867), a hypocretin receptor-1 antagonist, preferentially 
prevents ethanol seeking: comparison with natural reward seeking. Addict. Biol. doi: 10.1111/j.13691600.2012.00480.x. [Epub ahead of print].

Martin-Fardon, R., Zorrilla, E. P., Ciccocioppo, R., and Weiss, F. (2010). Role of innate and drug-induced dysregulation of brain stress and arousal systems in addiction: focus on corticotropin-releasing factor, nociceptin/orphanin FQ, and orexin/hypocretin. Brain Res. 1314, 145-161.

McFarland, K., and Kalivas, P. W. (2001). The circuitry mediating cocaine-induced reinstatement of drug-seeking behavior. J. Neurosci. 21, 8655-8663.

McLellan, A. T., Lewis, D. C., O’Brien, C. P., and Kleber, H. D. (2000). Drug dependence, a chronic medical illness: implications for treatment, insurance, and outcomes evaluation. JAMA 284, 1689-1695.

Miller, N. S., and Goldsmith, R. J. (2001). Craving for alcohol and drugs in animals and humans: biology and behavior. J. Addict. Dis. 20, 87-104.

Moussawi, K., Pacchioni, A., Moran, M., Olive, M. F., Gass, J. T., Lavin, A., et al. (2009). N-Acetylcysteine reverses cocaine-induced metaplasticity. Nat. Neurosci. 12, 182-189.

Nakahara, K., Fukui, K., and Murakami, N. (2004). Involvement of thalamic paraventricular nucleus in the anticipatory reaction under food restriction in the rat. J. Vet. Med. Sci. 66, 1297-1300.

O’Brien, C. P., Childress, A. R., Ehrman, R., and Robbins, S. J. (1998). Conditioning factors in drug abuse: can they explain compulsion? J. Psychopharmacol. 12, 15-22.

O'Brien, C. P., and McLellan, A. T. (1996). Myths about the treatment of addiction. Lancet 347, 237-240.
Otake, K. (2005). Cholecystokinin and substance $\mathrm{P}$ immunoreactive projections to the paraventricular thalamic nucleus in the rat. Neurosci. Res. 51, 383-394.

Parsons, M. P., Li, S., and Kirouac, G. J. (2006). The paraventricular nucleus of the thalamus as an interface between the orexin and CART peptides and the shell of the nucleus accumbens. Synapse 59, 480-490.

Pasumarthi, R. K., and Fadel, J. (2008). Activation of orexin/hypocretin projections to basal forebrain and paraventricular thalamus by acute nicotine. Brain Res. Bull. 77, 367-373.

Peyron, C., Tighe, D. K., Van Den Pol, A. N., De Lecea, L., Heller, H. C., Sutcliffe, J. G., et al. (1998). Neurons containing hypocretin (orexin) project to multiple neuronal systems. J. Neurosci. 18, 9996-10015.

Richards, J. K., Simms, J. A., Steensland, P., Taha, S. A., Borgland, S. L., Bonci, A., et al. (2008). Inhibition of orexin-1/hypocretin-1 receptors inhibits yohimbine-induced reinstatement of ethanol and sucrose seeking in Long-Evans rats. Psychopharmacology (Berl.) 199, 109-117.

Sakurai, T., Amemiya, A., Ishii, M., Matsuzaki, I., Chemelli, R. M., Tanaka, H., et al. (1998). Orexins and orexin receptors: a family of hypothalamic neuropeptides and $\mathrm{G}$ protein-coupled receptors that regulate feeding behavior. Cell 92, 573-585.

Sartor, G. C., and Aston-Jones, G. S. (2012). A septal-hypothalamic pathway drives orexin neurons, which is necessary for conditioned cocaine preference. J. Neurosci. 32, 4623-4631.

Scammell, T. E., and Winrow, C. J. (2011). Orexin receptors: pharmacology and therapeutic opportunities. Annu. Rev. Pharmacol. Toxicol. 51, 243-266.

See, R. E. (2002). Neural substrates of conditioned-cued relapse to drug-seeking behavior. Pharmacol. Biochem. Behav. 71, 517-529.

Sidhpura, N., Weiss, F., and MartinFardon, R. (2010). Effects of the mGlu2/3 agonist LY379268 and the mGlu5 antagonist MTEP on ethanol seeking and reinforcement are differentially altered in rats with a history of ethanol dependence. Biol. Psychiatry 67, 804-811.

Smith, R. J., Tahsili-Fahadan, P., and Aston-Jones, G. (2010) Orexin/hypocretin is necessary for context-driven cocaine-seeking. Neuropharmacology 58, 179-184.

Steketee, J. D., and Kalivas, P. W. (2011). Drug wanting: behavioral sensitization and relapse to drugseeking behavior. Pharmacol. Rev. 63, 348-365.

Sutcliffe, J. G., and de Lecea, L. (2002). The hypocretins: setting the arousal threshold. Nat. Rev. Neurosci. 3, 339-349.

Taheri, S., Zeitzer, J. M., and Mignot, E. (2002). The role of hypocretins (orexins) in sleep regulation and narcolepsy. Annu. Rev. Neurosci. 25, 283-313.

Teske, J. A., Billington, C. J., and Kotz, C. M. (2010). Hypocretin/orexin and energy expenditure. Acta Physiol. (Oxf.) 198, 303-312.

Thompson, J. L., and Borgland, S. L. (2011). A role for hypocretin/orexin in motivation. Behav Brain Res. 217, 446-453.

Van Der Werf, Y. D., Witter, M. P. and Groenewegen, H. J. (2002). The intralaminar and midline nuclei of the thalamus. Anatomical and functional evidence for participation in processes of arousal and awareness. Brain Res. Brain Res. Rev. 39, 107-140.

Wanat, M. J., Willuhn, I., Clark, J. J., and Phillips, P. E. (2009). Phasic dopamine release in appetitive behaviors and drug addiction. Curr. Drug Abuse Rev. 2, 195-213.

Weiss, F. (2005). Neurobiology of craving, conditioned reward and relapse. Curr. Opin. Pharmacol. 5, 9-19.

Willie, J. T., Chemelli, R. M., Sinton, C. M., and Yanagisawa, M. (2001). To eat or to sleep? Orexin in the regulation of feeding and wakefulness. Annu. Rev. Neurosci. 24, 429-458.

Winsky-Sommerer, R., Yamanaka, A., Diano, S., Borok, E., Roberts, A. J., Sakurai, T., et al. (2004). Interaction between the corticotropin-releasing factor system and hypocretins (orexins): a novel circuit mediating stress response. J. Neurosci. 24, 11439-11448.

Conflict of Interest Statement: The authors declare that the research was conducted in the absence of any commercial or financial relationships that could be construed as a potential conflict of interest.

Received: 16 August 2012; paper pending published: 13 September 2012; accepted: 19 October 2012; published online: 09 November 2012.

Citation: Martin-Fardon $R$ and Boutrel $B$ (2012) Orexin/hypocretin (Orx/Hcrt) transmission and drug-seeking behavior: is the paraventricular nucleus of the thalamus (PVT) part of the drug seeking circuitry? Front. Behav. Neurosci. 6:75. doi: 10.3389/fnbeh.2012.00075

Copyright (c) 2012 Martin-Fardon and Boutrel. This is an open-access article distributed under the terms of the Creative Commons Attribution License, which permits use, distribution and reproduction in other forums, provided the original authors and source are credited and subject to any copyright notices concerning any third-party graphics etc. 